\title{
Concept Software Based on Kinect for Assessing Dual-Task Ability of Elderly People
}

\section{AUTHOR(S):}

Kayama, Hiroki; Okamoto, Kazuya; Nishiguchi, Shu; Nagai, Koutatsu; Yamada, Minoru; Aoyama, Tomoki

\section{CITATION:}

Kayama, Hiroki ...[et al]. Concept Software Based on Kinect for Assessing Dual-Task Ability of Elderly People. Games for Health Journal 2012, 1(5): 348-352

\section{ISSUE DATE:}

2012-10-05

URL:

http://hdl.handle.net/2433/160130

\section{RIGHT:}

(C2012 Mary Ann Liebert, Inc.; "Games for Health Journal" is available online at: http://online.liebertpub.com.; This is not the published version. Please cite only the published version.; この論文は出版社版でありません。引用の際には出版社版をご確認 ご利用ください。 


\title{
Concept Software Based on Kinect for Assessing Dual-Task Ability of Elderly People
}

\author{
Hiroki Kayama, PT, Kazuya Okamoto,2 Shu Nishiguchi, PT, Koutatsu Nagai, PT, PhD, ${ }^{1}$ \\ Minoru Yamada, PT, PhD, and Tomoki Aoyama, MD, PhD ${ }^{1}$
}

\begin{abstract}
Objective: Assessment of fall risk of elderly people is a critical issue. Dual-task (DT) ability is a criterion for risk assessment. We developed new concept software based on Microsoft (Redmond, WA) Kinect ${ }^{\mathrm{TM}}$ for assessing DT ability. The software is named "Dual-Task Tai Chi" (DTTC) and includes Tai Chi and number place (Sudoku) components. The purpose of this study is to validate the DTTC test for assessment of DT ability.

Subjects and Methods: Forty-five community-dwelling elderly (mean age, $74.1 \pm 6.6$ years) individuals participated in this study. They performed DTTC, locomotive, cognitive, and DT tests. DT ability was evaluated with a $10-\mathrm{m}$ walk under a cognitive-task condition and a 10-m walk under a manual-task condition. The correlation between the time taken to complete the DTTC test and each function test was determined using Pearson correlation coefficients. Stepwise multiple regression analysis was conducted to assess the relationship between the DTTC test results and results of the other tests.

Results: The time taken to complete the DTTC test was correlated with DT ability, locomotive functioning, and cognitive functioning. Results of stepwise multiple regression analysis confirmed that DT, balance, and cognitive ability are statistically significant. No statistically significant association was found for the other variables. Conclusions: The DTTC test quantitatively evaluates a compound function including DT, balance, and cognitive abilities.
\end{abstract}

\section{Introduction}

$\mathbf{F}$ ALLS AMONG OLDER ADULTS are a serious problem in countries with large populations of older people. It has been estimated that $32 \%$ of community-dwelling individuals $\geq 75$ years old will fall at least once during a 1-year interval and that 24 percent of them will sustain serious injuries. ${ }^{1,2}$ Falling is, therefore, a common problem in the growing population of elderly people. The cost of treating fall-related injuries is substantial. ${ }^{3}$

Many researchers have studied the risk of falls in older people and countermeasures to prevent them. Motor functions (e.g., muscle force, balance ability, and gait performance) are an important factor known by the public. The next most important risk factor is reported to be cognitive impairment. ${ }^{4-6}$ Dual-task (DT) activity (i.e., performance of simultaneous locomotive and cognitive tasks) has recently become more important for assessment of fall risk. ${ }^{7}$ Improving DT performance can also help prevent falls in older people. ${ }^{8,9}$
While evaluating the locomotive, cognitive, and DT skills of the elderly and providing active intervention, it is vital to quantify these skills. There are many assessment methods and exercises for improving locomotive and cognitive performance to prevent falls, ${ }^{4-6}$ but there are few methods for evaluating simultaneous locomotive and cognitive performance (DT activity). We think that a more accessible measure of DT performance will facilitate interventions that are more effective for preventing falls in older people.

Commercial videogames such as the Nintendo (Redmond, WA) "Wii ${ }^{\mathrm{TM}} \mathrm{Fit}^{\prime}$ have generated much interest because of their promotion of health ${ }^{10}$ and rehabilitation. ${ }^{11}$ We previously reported that the scoring of a Nintendo "Wii Fit" program was associated with fall risk in community-dwelling older adults. ${ }^{12}$ We also developed a smartphone application for assessment of fall risk. ${ }^{13}$ However, motion-tracking controllers such as the Nintendo Wiimote are not sensitive enough to accurately interpret the user's movements, and the smartphone tool is limited to assessing the motion of only one body segment.

\footnotetext{
${ }^{1}$ Department of Physical Therapy, Human Health Sciences, Graduate School of Medicine, Kyoto University, Kyoto, Japan.

${ }^{2}$ Division of Medical Information Technology and Administration Planning, Kyoto University Hospital, Kyoto, Japan.

${ }^{3}$ Faculty of Health Science, Department of Physical Therapy, Kyoto Tachibana University, Kyoto, Japan.
} 
The Kinect ${ }^{\mathrm{TM}}$ sensor (Microsoft, Redmond) does not require the user to hold an interface device. The sensor is a lowcost, depth-sensing camera (produced by the Israeli company PrimeSense $^{\circledR}$ [Tel Aviv]) that captures the user's full-body movements in three-dimensional space and incorporates them in the game. The user's body, operating in threedimensional space, replaces traditional handheld controllers. The Kinect camera can be connected to a personal computer. The Kinect system is flexible and commercially available.

We developed a new concept device to assess DT ability and named it the "Dual-Task Tai Chi" (DTTC) test. ${ }^{14}$ To quantify DT skill, we developed this system using Kinect, a motion-capture device. We chose Tai Chi as a locomotive task and Sudoku as a cognitive task. In the input and convert module of the game system, Kinect translates positional data of the user and objects located in front of the system into an animated stick figure, which is displayed on a screen. A Sudoku is simultaneously displayed on a screen.

A reason for combining Tai Chi exercises with Sudoku is that we can change the level of difficulty and guide the subject's movements by changing the position of empty boxes in the Sudoku puzzle. Additionally, Tai Chi is a valid locomotive task based on full-body motion, and Tai Chi training is useful for preventing falls in elderly people. ${ }^{15}$ Sudoku is a useful tool for assessing cognitive ability. Sudoku exercises improve cognitive performance (specifically, reaction time and number of correct and missing answers) of people with Parkinson's disease, as measured by the Stroop test. ${ }^{6}$ The purpose of this study was to validate this new concept device as an assessment tool.

\section{Subjects and Methods}

\section{Participants}

Forty-five community-dwelling, elderly subjects participated in this study. They were recruited by means of an advertisement in the local press. The following selection criteria were used: age $\geq 60$ years, community-dwelling, independent ambulation, willingness to participate in the measurement of physical fitness, and minimal hearing and vision impairments. Exclusion criteria were as follows: inability to complete the tasks because of reduced cognitive function; severe cardiac, pulmonary, or musculoskeletal disorders; pathologies associated with increased risk of falls, such as Parkinson's disease or stroke; osteoporosis; and use of psychotropic drugs. We obtained informed consent from each participant. This study was approved (protocol approval E880) by the Ethical Review Board of Kyoto University Graduate School of Medicine, Kyoto, Japan.

\section{Measurement device}

The DTTC test requires users to solve a number place problem (Sudoku) by controlling a stick figure with movement of their entire body. The user's full-body motion is captured using Kinect, a motion-capture device, and is translated into movements of a stick figure on a screen. The cognitive task is to fill in three boxes chosen randomly from a $4 \times 4$ grid with digits ranging from 1 to 4 . The user selects a digit using his or her right hand and left foot and points to a box with his or her left hand. In addition, the user must move his or her right hand to the left hand to fill the pointed box with the selected digit. As such, a full-body motion similar to Tai Chi Chuan movements is required. We recorded the time taken to fill in all three boxes; this was our evaluation index.

To begin with, the user stands $3 \mathrm{~m}$ in front of the Kinect sensor with his or her right foot in front of the sensor (Fig. 1). The following instructions were provided (Fig. 2):

1. Reach a digit you need to answer with your right hand.

2. Step $50 \mathrm{~cm}$ laterally with your left leg to grip a number in your right hand.

3. Select a blank you want to answer with your left hand, and move your right hand to your left one.

A pilot experiment indicated that the test-retest reliability for completion time was considerably high (inter-trial correlation coefficient, $0.94 ; 95 \%$ confidence interval, $0.86-0.97$; $P<0.001){ }^{14}$

\section{Cognitive status measures}

Cognitive function was evaluated with the Rapid Dementia Screening Test (RDST) ${ }_{1}^{16}$ trail-making test (TMT) ${ }_{1}^{17}$ and verbal fluency test (VFT) ${ }^{18-20}$ The TMT is a wellestablished psychomotor test originally developed as part of the Army Individual Test Battery ${ }^{17}$ and has been widely used in clinical evaluations to assess deficits in executive cognitive function. The VFT has a letter fluency component and a category fluency component. ${ }^{17}$ Participants were asked to think of as many animal names as possible in 1 minute (category fluency). ${ }^{19}$ Verbal fluency is an evaluation of expressive language ability and executive function. ${ }^{18-20}$ The score was the number of successful words (except for some proper nouns).

\section{Physical performance measures}

Locomotive function was evaluated as 10-m walking time under standard conditions (ST walking), ${ }^{21}$ 10-m maximum walking time (10-m MAX) (see DT performance measures), functional reach (FR), ${ }^{22}$ timed up-and-go test (TUG), ${ }^{23}$ oneleg standing (OLS) test, ${ }^{24}$ and five-chair stand (5-CS) test. ${ }^{25}$ In the ST walking test, participants were asked to walk $15 \mathrm{~m}$ at a speed that was comfortable to them, and the time taken to

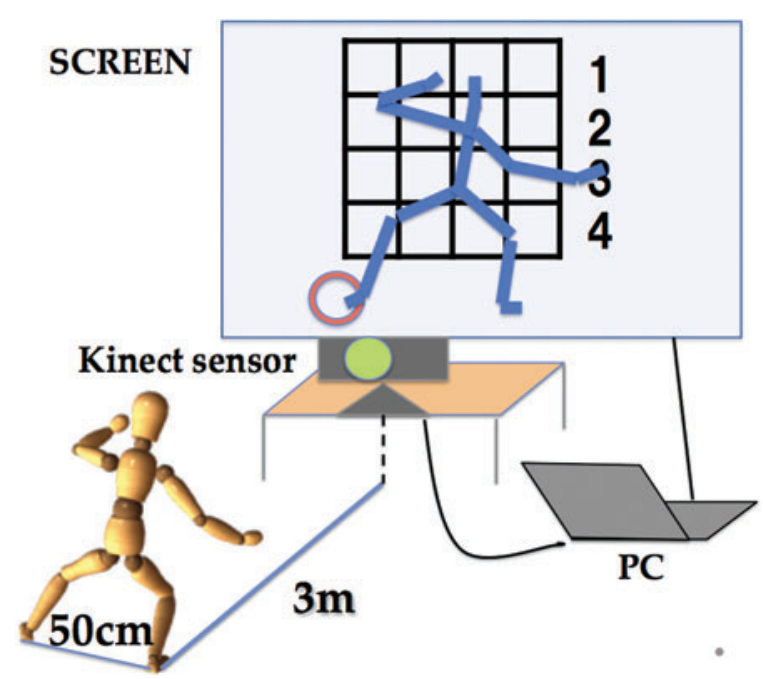

FIG. 1. Big-picture view of Dual-Task Tai Chi test. Color images available online at www.liebertpub.com/g4h 


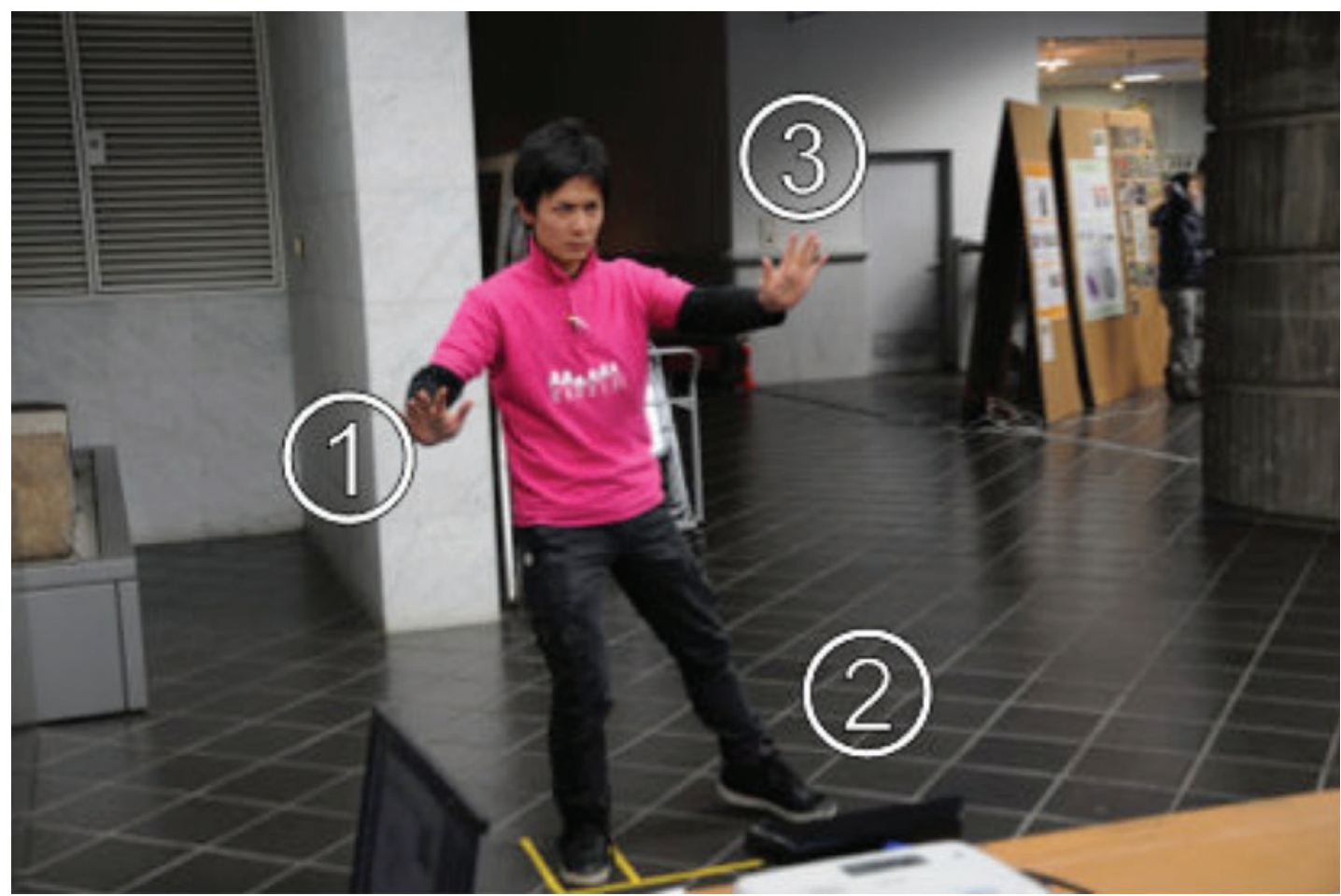

FIG. 2. Numbered instructions for the Dual-Task Tai Chi test (see text). Color images available online at www .liebertpub.com/g4h

walk $10 \mathrm{~m}$ during this walk was measured using a stopwatch. The ST walking score was the average time recorded for two trials. In the FR test, each participant was positioned next to a wall, with one arm raised at $90^{\circ}$ and fingers extended. A yardstick was mounted on the wall at shoulder height. The distance the participant could reach while extending forward from an initial upright posture to the maximal anterior leaning posture without moving or lifting the feet was visually measured in centimeters as the third fingertip position against the mounted yardstick. The distances measured in two trials were averaged to obtain the FR score. In the TUG test, participants were asked to stand up from a standard chair with a seat height of $40 \mathrm{~cm}$, walk a distance of $3 \mathrm{~m}$ at a normal pace, turn, walk back to the chair, and sit down. The time recorded in two trials was averaged to obtain the TUG score. In the OLS test, participants were instructed to start from a position with a comfortable base as support, with eyes open and arms along the side of the trunk. They were then instructed to stand unassisted on either leg. The OLS time was the number of seconds from when one foot was lifted from the floor to when it touched the floor again or the standing leg. In the 5-CS test, participants were asked to stand up and sit down five times as quickly as possible. They were timed from the initial sitting position to the final standing position, at the end of the fifth stand.

\section{DT performance measures}

A 10-m walk under a cognitive-task condition (CT walking) and a 10-m walk under a manual-task condition (MT walking) were used to evaluate DT function..$^{8,9}$ The method for measuring 10-m MAX, CT walking, and MT walking is roughly the same as the method for the ST walking test. For 10-m MAX, we asked participants to walk as fast as possible. For CT walking, we asked them to walk at the most comfortable speed while counting down from 100. For MT walking, we asked them to carry a ball $(7 \mathrm{~cm}$ in diameter, $150 \mathrm{~g})$ on a tray $(17 \mathrm{~cm}$ in diameter, $50 \mathrm{~g})$ while walking at the most comfortable speed.

\section{Statistical analysis}

The correlation between the time taken to complete the DTTC and functional tests was determined using Pearson correlation coefficient. Additionally, we evaluated the association between DTTC time and other test results using stepwise multiple regression analysis. We included DTTC time as the dependent variable and age, ST walking, MT walking, TUG, FR, OLS, 5-CS, TMT-B, RDST, and VFT as independent variables. We considered multicollinearities among tests when we chose these variables. A value of $P<0.05$ was considered statistically significant for all analyses.

\section{Results}

\section{Subject characteristics}

The 45 subjects were $60-91$ years old, with a mean age of $74.1 \pm 6.6$ years; many subjects were in their $70 \mathrm{~s}$. The male-tofemale ratio was 1:4. All the participants completed the DTTC program. The mean time taken to complete the DTTC test was $50.2 \pm 27.1$ seconds.

\section{Correlation analysis}

Table 1 shows the Pearson correlation coefficients for time to complete the DTTC program and other functional tests for 
Table 1. Pearson Correlation Coefficients of the Time Taken to Complete the “Dual-Task Tai Chi”

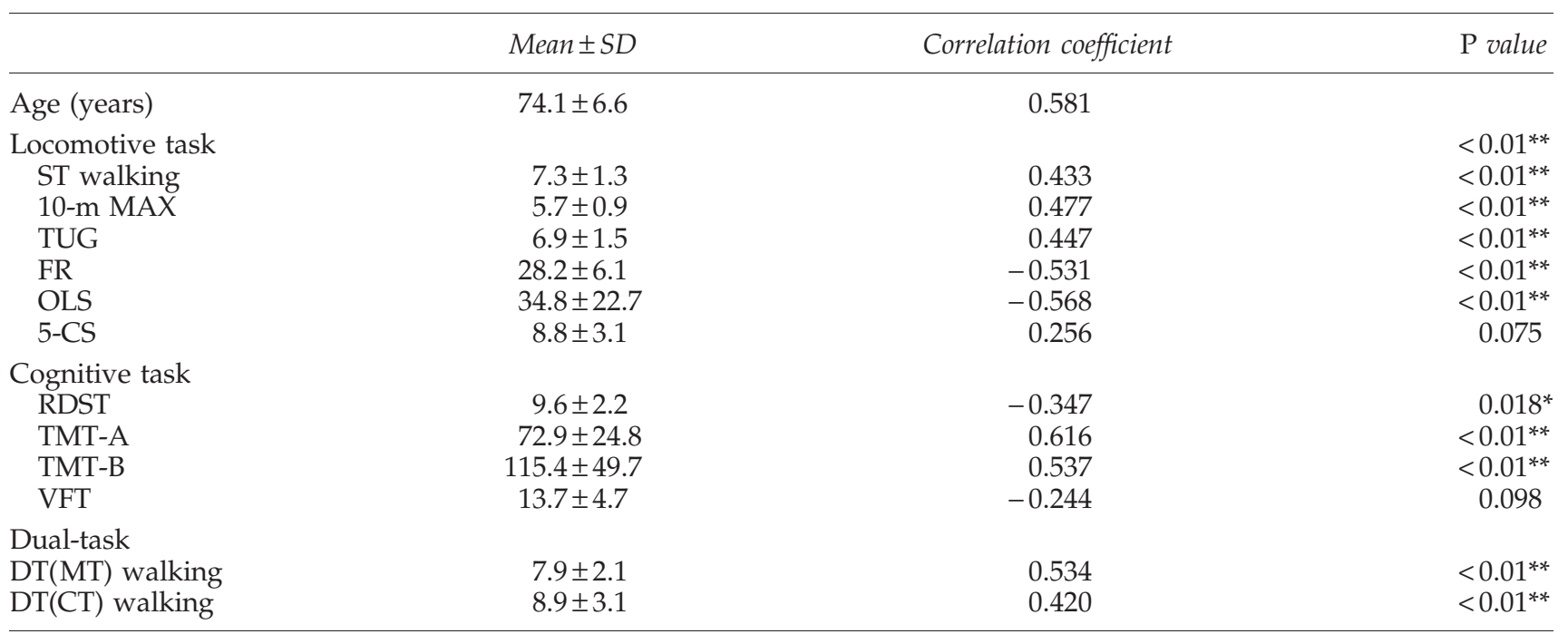

${ }^{*} P<0.05$, significant difference; ${ }^{* *} P<0.01$, highly significant difference.

5-CS, five-chair stand test; 10-m MAX=10-m maximal walking time; DT(CT) walking, 10-m walk under a cognitive-task condition DT(MT) walking, 10-m walk under a manual-task condition; FR, functional reach; OLS, one-leg standings; RDST, Rapid Dementia Screening Test; ST walking, 10-m walking time under standard conditions; TMT, trail-making test; TUG, timed up-and-go test; VFL, verbal fluency test.

DT, locomotive, and cognitive performance. Time was strongly correlated not only with the DT function test but also with locomotive function and cognitive function tests $(P<0.05)$.

Results of stepwise multiple regression analysis confirmed that MT walking, FR, OLS, and TMT-B were statistically significant $(P<0.05)$. No statistically significant association was found for the other variables (Table 2).

\section{Discussion}

This study has two main components. First, the purpose of the new device is to evaluate DT ability, comprising a cognitive task and a locomotive task. It is difficult to quantify locomotive skill. In general, locomotive function is evaluated using devices to capture motion and measure ground reaction forces and triaxial acceleration. ${ }^{26}$ The next task was to combine quantifications of locomotive and cognitive skills.

Table 2. Stepwise Multiple Regression Analysis for the Time Taken to Complete the "Dual-Task Tai Chi"

\begin{tabular}{lcc}
\hline Independent variable & $\beta$ estimates & P value \\
\hline OLS & -0.392 & $<0.01^{* *}$ \\
FR & -0.302 & $<0.01^{* *}$ \\
DT(MT) walking & 0.276 & $0.049^{*}$ \\
TMT-B & 0.269 & $0.046^{*}$ \\
ST walking & - & - \\
TUG & - & - \\
5-CS & - & - \\
RDST & - & - \\
VFT & - & - \\
\hline
\end{tabular}

${ }^{*} P<0.05$, significant difference; ${ }^{* *} P<0.01$, highly significan difference.

5-CS, five-chair stand test; DT(MT) walking, 10-m walk under a manual-task condition; FR, functional reach; OLS, one-leg standings; RDST, Rapid Dementia Screening Test; ST walking, 10-m walking time under standard conditions; TMT, trail-making test; TUG, timed up-and-go test; VFL, verbal fluency test.
We required a motion-capture device capable of accurately capturing full-body motions. Kinect is an innovative motioncapture device, and its software development kit enabled us to capture a user's motions while the user performed a cognitive task in real time. We designed the DTTC to mimic Tai Chi Chuan movements (a motor task) and to include Sudoku number place problems (a cognitive task). It is difficult to fill the boxes by full-body motions while balancing, let alone while solving Sudoku. Because the degree of difficulty of the locomotive, cognitive, and dual tasks was moderate, we were able to obtain results. The strong correlation between DTTC time and the practical assessment of DT suggests that the DTTC is a useful device for assessing DT ability. A great advantage of Kinect is that there is no need to use controllers or markers. Therefore, movement is not restricted, and the exercise is quite exciting. Kinect holds promise not only for promoting health but also for rehabilitation of older adults.

Second, Kinect is a promising augmented-reality game system. This technology opens up new possibilities. For instance, it has been used to train surgeons by providing threedimensional information, and now it is being used for rehabilitation. ${ }^{27,28}$ Commercial augmented-reality game "Wii Fit" (Nintendo) software has already been used for rehabilitation. ${ }^{10-12}$ Adaptability to acute environmental change is a major health issue for elderly Japanese individuals, as it involves locomotive and cognitive function. Some individuals may isolate themselves, withdrawing from social activity, because they cannot adapt to environmental changes. They confuse real and virtual space. Augmented-reality technology will continue to develop and mix real and virtual space for our amusement or to make our daily life easier, but this could be chaotic for some elderly people. We hope that new augmented-reality devices like the DTTC will be used to help elderly people improve physical function and adaptability and will motivate them to remain socially active.

The current study has several limitations. First, the number of participants was small, and physical ability varied greatly according to age and according to the individual. Second, this 
study showed the accuracy of the DTTC test as an assessment of DT ability. The main purpose of this system is to assess fall risk. A large cross-sectional or longitudinal study is required to evaluate the reliability and effectiveness of the DTTC test. Moreover, an intervention study is required to determine the usefulness of DTTC for preventing falls. DTTC may be a promising game system for promoting health.

\section{Acknowledgments}

The authors thank the participants and thank our department for helpful advice.

\section{Author Disclosure Statement}

No competing financial interests exist.

\section{References}

1. Tinetti ME, Speechley M. Prevention of falls among the elderly. N Engl J Med 1989; 320:1055-1059.

2. Tinetti ME, Speechley M, Ginter SF. Risk factors for falls among elderly persons living in the community. $\mathrm{N}$ Engl J Med 1988; 319:1701-1707.

3. Scuffhum $P$, Chaplin S, Legood R. Incidence and costs of unintentional falls in older people in the United Kingdom. J Epidemiol Community Health 2003; 57:740-744.

4. Guidelines for the prevention of falls in older persons. American Geriatrics Society, British Geriatrics Society, and the American Academy of Orthopedic Surgeons Panel on Falls Prevention. J Am Geriatr Soc 2001; 49:664-672.

5. Muir SW, Gopaul K, Montero Odasso MM. The role of cognitive impairment in fall risk among older adults: A systematic review and meta-analysis. Age Ageing 2012; 41:299-308.

6. Nombela C, Bustillo PJ, Castell PF, et al. Cognitive rehabilitation in Parkinson's disease: Evidence from neuroimaging. Front Neurol 2011; 2:82.

7. Lundin-Olsson L, Nyberg L, Gustafson Y. Attention, frailty, and falls: The effect of a manual task on basic mobility. J Am Geriatr Soc 1998; 46:758-761.

8. Silsupado P. Effects of single-task versus dual-task training on balance performance in older adults: A double blind randomized controlled trial. Arch Phys Med Rehabil 2009; 90:381-387.

9. Yamada M, Aoyama T, Arai H, et al. Dual-task walk is a reliable predictor of falls in robust elderly adults. J Am Geriatr Soc 2011; 59:163-164.

10. Worley JR, Rogers SN, Kraemer RR. Metabolic responses to Wii Fit ${ }^{\mathrm{TM}}$ video games at different game levels. J Strength Cond Res 2011; 25:689-693.

11. Chan TC, Chan F, Shea YF, et al. Interactive virtual reality Wii in geriatric day hospital: A study to assess its feasibility, acceptability and efficacy. Geriatr Gerontol Int 2012 Apr 2 [Epub ahead of print]. doi: 10.1111/j.1447-0594.2012.00848.x.

12. Yamada M, Aoyama T, Ichihashi N, et al. The reliability and preliminary validity of game-based fall risk assessment in community-dwelling older adults. Geriatr Nurs 2011; 32:188-194.

13. Yamada M, Aoyama T, Okamoto K, et al. Using a smartphone while walking: A measure of dual-tasking ability as a falls risk assessment tool. Age Ageing 2011; 40:516-519.
14. Okamoto K, Kayama H, Yamada M, et al. The development of a system for the assessment of a dual-task performance based on a motion-capture device. Proc ICDVRAT 2012 (in press).

15. Leung DP, Chan CK, Tsang HW, et al. Tai Chi as an intervention to improve balance and reduce falls in older adults: A systematic and meta-analytical review. Altern Ther Health Med 2011; 17:40-48.

16. Kalbe E, Calabrese P, Schwalen S, Kessler J. The Rapid Dementia Screening Test (RDST): A new economical tool for detecting possible patients with dementia. Dement Geriatr Cogn Disord 2003; 16:193-199.

17. War Department Adjutant General's Office. Army Individual Test Battery. Manual of Directions and Scoring. Washington, DC: War Department, Adjutant General's Office; 1944.

18. Benton AL. Differential behavioral effects in frontal lobe disease. Neuropsychologia 1968; 6:53-60.

19. Nemoto T, Kashima H, Mizuno M. Contribution of divergent thinking to community functioning in schizophrenia. Prog Neuropsychopharmacol Biol Psychiatry 2007; 31:517524.

20. Lezak MD. Neuropsychological Assessment, 4th ed. New York: Oxford University Press; 2004.

21. Lopopolo RB, Greco M, Sullivan D, et al. Effect of therapeutic exercise on gait speed in community-dwelling elderly people: A meta-analysis. Phys Ther 2006; 86:520-540.

22. Duncan PW, Weiner DK, Chandler J, Studenski S. Functional reach: A new clinical measure of balance. J Gerontol 1990; 45:M192-M197.

23. Podsiadlo D, Richardson S. The timed "Up \& Go": A test of basic functional mobility for frail elderly persons. J Am Geriatr Soc 1991; 39:142-148.

24. Vellas BJ, Wayne SJ, Romero L, et al. One-leg balance is an important predictor of injurious falls in older persons. J Am Geriatr Soc 1997; 45:735-738.

25. Guralnik JM, Simonsick EM, Ferrucci L, et al. A short physical performance battery assessing lower extremity function: Association with self-reported disability and prediction of mortality and nursing home admission. J Gerontol 1994; 49:M85-M94.

26. Reid M, Whiteside D, Elliott B. Serving to different locations: Set-up, toss, and racket kinematics of the professional tennis serve. Sports Biomech 2011; 10:407-414.

27. Shenai MB, Dillavou M, Shum C, et al. Virtual interactive presence and augmented reality (VIPAR) for remote surgical assistance. Neurosurgery 2011; 68:200-207.

28. Espay AJ, Baram Y, Dwivedi AK, et al. At-home training with closed-loop augmented-reality cueing device for improving gait in patients with Parkinson disease. J Rehabil Res Dev 2010; 47:573-581.

$$
\begin{array}{r}
\text { Address correspondence to: } \\
\text { Hiroki Kayama, PT } \\
\text { Department of Physical Therapy } \\
\text { Human Health Sciences } \\
\text { Graduate School of Medicine } \\
\text { Kyoto University } \\
\text { 53, Kawahara-cho, Shogoin, Sakyo-ku } \\
\text { Kyoto 606-8507, Japan }
\end{array}
$$

E-mail: kayama.hiroki.37r@st.kyoto-u.ac.jp 


\section{AUTHOR QUERY FOR G4H-2012-0019-VER9-KAYAMA_1P}

AU1: Provide academic degree for this co-author.

AU2: Addition ok for 10-m MAX definition? All other terms are defined below.

AU3: Update with volume and pages, or give doi assigned by publisher.

AU4: Differences as meant?

AU5: Differences as meant? 